\title{
A LARGE INTRAHEPATIC PORTAL VEIN ANEURYSM: A CASE REPORT
}

Manoj Hazarika ${ }^{1}$, Nabanita Deka² ${ }^{2}$ Gautam Goswami ${ }^{3}$

\section{HOW TO CITE THIS ARTICLE:}

Manoj Hazarika, Nabanita Deka, Gautam Goswami. "A Large Intrahepatic Portal Vein Aneurysm: A Case Report". Journal of Evolution of Medical and Dental Sciences 2014; Vol. 3, Issue 49, October 02; Page: 11662-11666, DOI: $10.14260 /$ jemds/2014/3532

ABSTRACT: Portal vein aneurysms are extremely rare and intrahepatic variety is the rarest. It is often an incidental, asymptomatic finding or may be associated with portal hypertension. Recognition of this finding can help to avoid potential confusion with abdominal masses of other aetiologies. In this report, we present a case of large intrahepatic portal vein aneurysm measuring approximately $7.7 \times 6.4 \mathrm{~cm}$. till date this is the largest intrahepatic portal vein aneurysm ever reported.

KEYWORDS: Aneurysm, intrahepatic portal vein.

CASE REPORT: A 25 years female with history of chronic vague abdominal discomfort was presented to the gastroenterology OPD. Per abdominal examination showed evidence of splenomegaly. There was no previous history of any surgical intervention or abdominal trauma. Liver function test showed elevation of hepatic enzymes. Complete haemogram and renal function test were within normal limits. She was advised a multiphasic CT abdomen to exclude any other abdominal pathologies. Multiphasic MDCT abdomen revealed a hypo dense lesion measuring approx $7.7 \mathrm{~cm} \times 6.4 \mathrm{~cm}$ in size predominantly occupying the left lobe of the liver.

No enhancement of the lesion is seen in the arterial phase. The lesion showed homogenous enhancement in the portal venous phase with branches of portal vein merging with the lesion suggesting portal venous origin of the lesion. The intrahepatic portal vein branches were otherwise ectatic. The main portal vein was also dilated and measures $18.5 \mathrm{~mm}$ near the splenoportal confluence. No thrombus is seen in the splenoportal axis. There was volume redistribution in the liver with enlargement of the left lobe and atrophy of the right lobe. No other focal lesion in the liver was seen. The paraumbilical vein was re canalised.

The intrahepatic bile ducts, CHD, CBD and intrahepatic veins were unremarkable. The spleen was grossly enlarged. The splenic vein was also dilated and measures approx $16.6 \mathrm{~mm}$. Superior mesenteric vein was normal. There was mild ascites. Pancreas was normal in size, shape and attenuation. No lymphadenopathy or any other abdominal pathology was seen. Constellation of findings suggested a saccular intrahepatic portal venous aneurysm with features of portal hypertension.

DISCUSSION: Aneurysms of the portal vein is extremely rare and represent only 3\% of all aneurysms of the venous system. ${ }^{1}$ Barzilai and Kleckner ${ }^{2}$ were the first to report a portal vein aneurysm, in 1956. The authors described the necropsy of a cirrhotic patient with a portal vein aneurysm and thrombus that ruptured into the biliary system. ${ }^{2}$

Portal vein aneurysms are defined as focal dilatation of portal venous system, either extrahepatic or intrahepatic, extrahepatic being more common. Though exact mechanism is unknown, various etiologies ranging from congenitally defective regression of right primitive 
vitelline vein to acquired causes such as portal hypertension, liver cirrhosis, trauma, pancreatitis, liver biopsy or tumor invasion have been reported. ${ }^{3-6}$

The portal venous system develops from the vitelline and umbilical veins that drain the intestinal blood of the embryo. It has been postulated that during embryologic development, there is a regression failure in the right primitive vitelline vein.

The diverticular remnant of the vitelline vein is believed to enlarge, forming a saccular aneurysm of the portal vein later in life. ${ }^{7}$ It is believed that the increase in intraluminal pressure observed in portal hypertension may lead to dilation of the relatively thin wall of the portal vein. However, the explanation for the significant disproportion between the incidence of portal hypertension and portal vein aneurysms suggests that there must be another associated cause. ${ }^{8}$

Aneurysms of the intrahepatic portal vein branches tend to be smaller than extrahepatic aneurysms, possibly due to the restrictive effects of the liver parenchyma on intrahepatic aneurysm growth.

Accordingly, extrahepatic portal venous dilatation is considered to be "aneurysmal" after exceeding $2 \mathrm{~cm}$ in diameter, while intrahepatic portal vein branches are considered to be "aneurysmal" when they exceed $0.7 \mathrm{~cm}$ in diameter in patients without relevant risk factors and 0.85 $\mathrm{cm}$ in diameter in cirrhotic patients. ${ }^{9}$

The most common location of portal vein aneurysm is at the splenomesenteric venous confluence, followed by the portal vein and its ramifications. ${ }^{10}$ Most portal venous system aneurysms are asymptomatic and do not demonstrate a significant increase in size once discovered, although some manifest with nonspecific abdominal pain as a major symptom.

Complications of portal venous system aneurysms are thrombosis, portal hypertension (due to flow alterations or compression of the main portal vein), rupture, thrombosis and distal embolism; compression of the common bile duct causing jaundice, cholestasis, and cholelithiasis; and compression of the duodenum. ${ }^{11}$

The management of portal vein aneurysms remains somewhat controversial. Asymptomatic, small aneurysms in patients without portal hypertension or cirrhosis are often managed conservatively with regular follow-ups of aneurysm size and monitoring for new symptoms.

Surgical intervention should, however, be considered if the portal vein aneurysm causes symptoms, or if the aneurysm expands and/or presents a high risk of thrombosis or rupture. Patients with portal hypertension and portal vein thrombosis have generally been candidates for shunt surgery. ${ }^{12}$

Our patient had a large intrahepatic portal vein aneurysm $7.7 \times 6.4 \mathrm{~cm}$ which is the largest reported case in the world till date from the available literatures. Previous largest intrahepatic aneurysm of size $5 \times 4 \mathrm{~cm}$ was reported by Kaido $\mathrm{T}$ at el, $2005 .{ }^{13}$

There was association of portal hypertension in this case. This patient is on conservative management and periodic follow up. 


\section{CASE REPORT}

Fig 1: (A) Multiphasic MDCT axial image shows no enhancement of the aneurysm (white arrow) in arterial phase. (B) The aneurysm vividly enhances in portal venous phase suggesting venous origin of the aneurysm (white arrow).

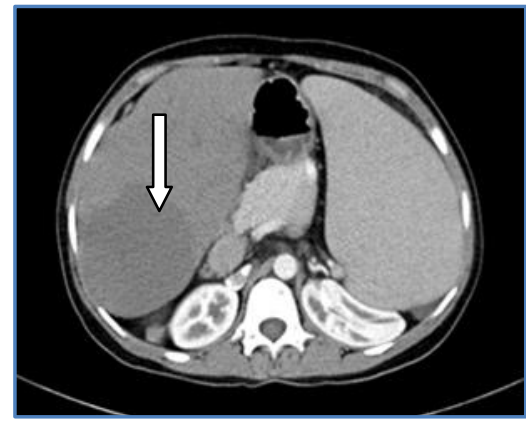

Fig. 1A

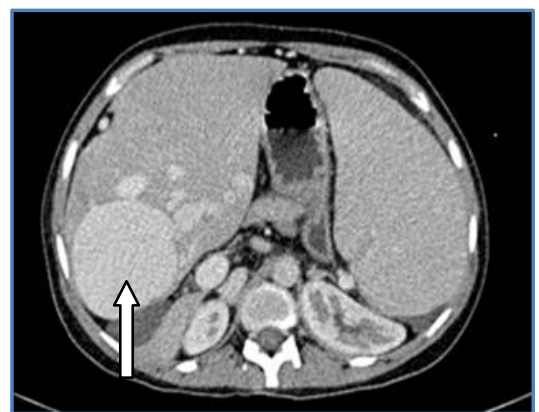

Fig. $1 B$

Fig. 2: The size of the aneurysm is shown in axial portal venous phase (A). Axial image in (B) reveals ectatic intrahepatic portal veins in the left lobe of the liver (thin white arrow). The small right lobe of the liver (thick white arrow) is shown along with the GB (curved arrow).

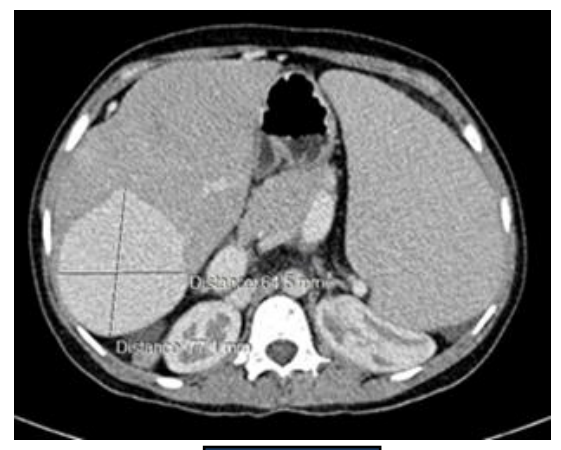

Fig. 2A

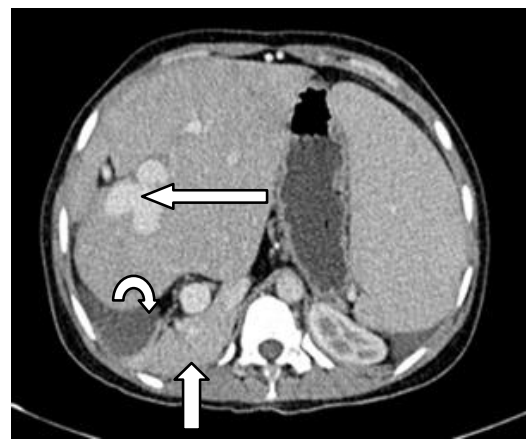

Fig. 2B

Fig 3: MDCT axial mages in portal venous phase in (A) and (B) showing dilated splenoportal confluence (thin black arrow), recanalised paraumbilical vein (white arrow) and splenomegaly (thick white arrow).

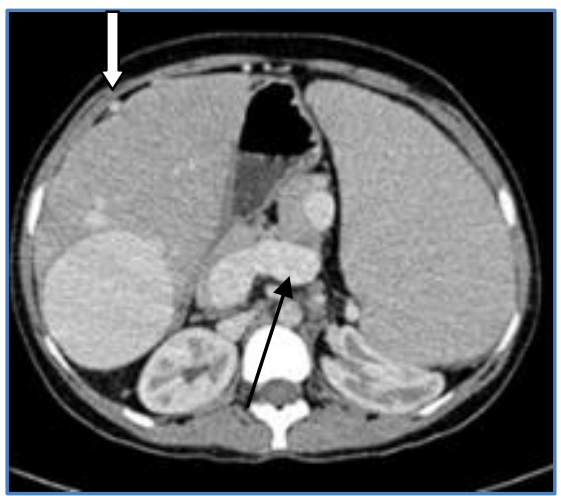

Fig. 3A

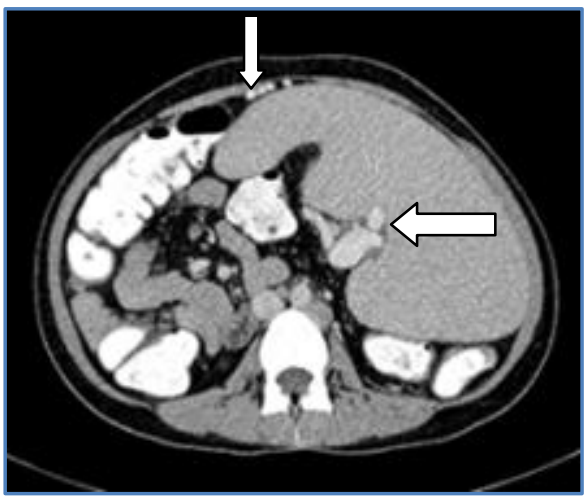

Fig. 3B 
Fig. 4: Coronal images in (A) and (B) showing intrahepatic portal venous aneurysm (thin black arrows). There is gross splenomegaly (thick white arrow) which is reaching upto left iliac fossa. The aneurysm (thin black arrow) is also shown in reformatted sagittal image in (C).

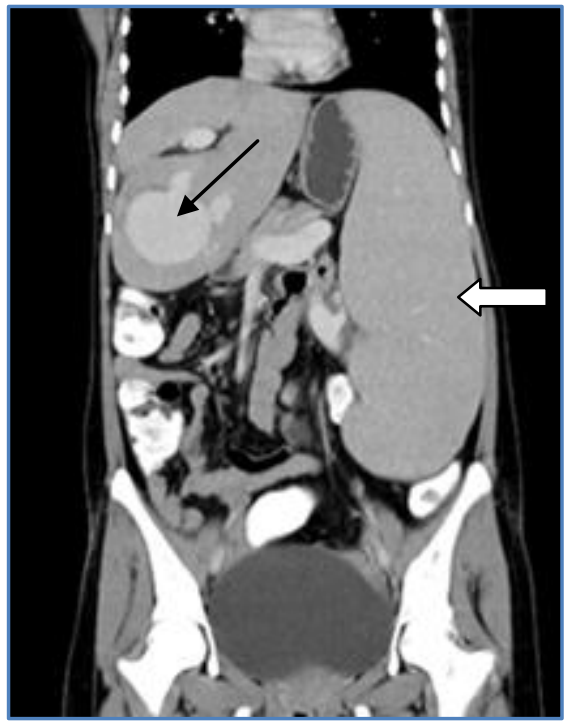

Fig. 4A

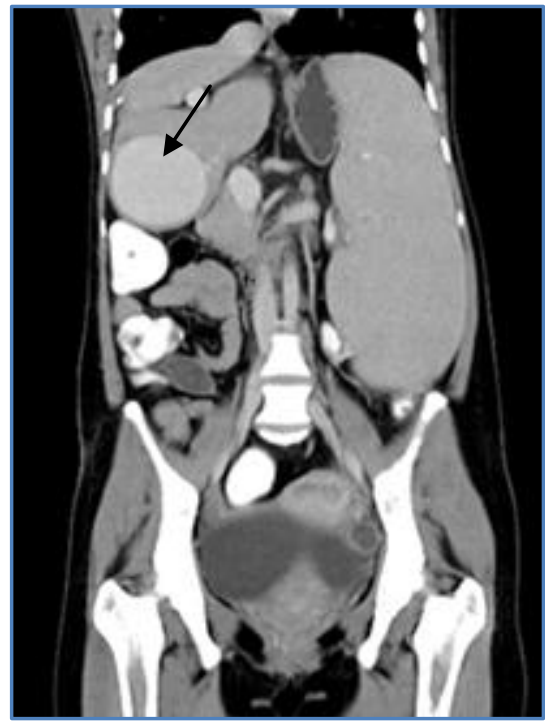

Fig. 4B

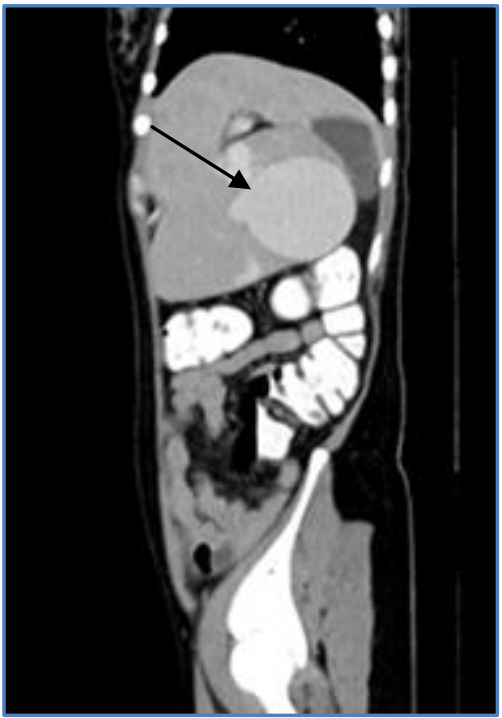

Fig. 4C

\section{REFERENCES:}

1. Andraus W, Amico E C, Machado M A. et al: portal vein aneurysm; Clinics 62(2) São Paulo 2007.

2. Barzilai R, Kleckner MS. Hemocholecyst following ruptured aneurysm of portal vein; report of a case. AMA Arch Surg. 1956; 72:725-7.

3. Ozbek SS, Killi MR, Pourbagher MA. Portal venous system aneurysms: report of five cases. J Ultrasound Med 1999; 18: 417-22.

4. Ryan B. Schwope, Daniel J. Margolis, Steven S. Raman, Barbara M. Kadell. Portal Vein Aneurysms: A Case Series with Literature Review. Radiology Case 2010; 4 (6):28-38.

5. Gallagher DM, Leiman S, Hux CH. In utero diagnosis of a portal vein aneurysm. J Ultrasound Med 1993; 21:147-51.

6. Yang DM, Yoon MH, Kim HS, Jin W, Hwang HY, Kim HS. CT findings of portal vein aneurysm caused by gastric adenocarcinoma invading the portal vein.Br J Radiol 2001;74(883):654-56

7. Lau H, Chew DK, Belkin M. Extrahepatic portal vein aneurysm: a case report and review of the literature. Cardiovasc Surg. 2002;10: 58-61.

8. Ohnishi K, Nakayama T, Saito M, Nomura F, Koen H, Tamaru J, et al. Aneurysm of the intrahepatic branch of the portal vein. Report of two cases. Gastroenterology 1984; 86:169-73.

9. Portal Vein Aneurysms: Imaging Manifestations and Clinical Significance Dalal PS, Raman SP, Horton KM, Fishman EK Emerg Radiol 2013 May 23.

10. Lopez-Machado E, Mallorquin-Jimenez F, Medina -Benitez A, Ruiz-Carazo E, Cubero-Garcia M. Aneurysms of the portal venous system: ultrasonography and CT findings. Euro J Radiol. 1998; 26: 210-4. 


\section{CASE REPORT}

11. Gallego C et al. Congenital and Acquired Anomalies of the Portal Venous System; Radio Graphics 2002; 22:141-159

12. Shazia A. Rafiq, MD and Michael D. Sitrin, MD. Portal Vein Aneurysm Case Report and Review of the Literature; Gastroenterol Hepatol (N Y). Apr 2007; 3(4): 296-298. PMCID: PMC3099279

13. Kaido T, Taii A, Nakajima T. A huge intrahepatic portal vein aneurysm; Abdom Imaging. 2005 Jan-Feb;30(1):69-70

\section{AUTHORS:}

1. Manoj Hazarika

2. Nabanita Deka

3. Gautam Goswami

\section{PARTICULARS OF CONTRIBUTORS:}

1. Assistant Professor, Department of Radiology, Gauhati Medical College \& Hospital, Guwahati, Assam, India.

2. Assistant Professor, Department of Radiology, Gauhati Medical College \& Hospital, Guwahati, Assam, India.

3. Professor and HOD, Department of Radiology, Gauhati Medical College \& Hospital, Guwahati, Assam, India.

\section{NAME ADDRESS EMAIL ID OF THE CORRESPONDING AUTHOR:}

Dr. Manoj Hazarika,

Assistant Professor,

Department of Radiology,

Gauhati Medical College \&

Hospital, Guwahati-781032,

Assam, India.

Email:manojhazarika23@gmail.com

Date of Submission: 14/09/2014.

Date of Peer Review: 15/09/2014.

Date of Acceptance: 25/09/2014.

Date of Publishing: 30/09/2014. 